\title{
WORKSHOP
}

\section{PENSION FUNDING WITH TIME DELAYS AND THE OPTIMAL SPREAD PERIOD}

\author{
By Steven Haberman \\ Department of Actuarial Science and Statistics \\ The City University, London, UK
}

\begin{abstract}
The paper extends earlier results by demonstrating that there is an optimal range of values for the period for amortizing valuation surpluses or deficiencies, in the case when there is a one year time delay between fixing a contribution rate and the accounting information about current fund levels. The optimal range is compared for the cases where there is no time delay and there is a one year time delay.
\end{abstract}

\section{KEYWORDS}

Pension funding; time delays; optimal spread period.

\section{INTRODUCTION}

We shall consider the financial structure of a defined benefit pension scheme, as represented by a simple mathematical model, which can be regarded as an extension to that originally proposed by Trowbridge (1952). We focus on the effect of varying investment returns on the contribution rate and fund level for the scheme and consider possible choices of two important control parameters: the spread period and the delay in fixing contribution rates.

We consider defined benefit pension schemes where the benefits promised in the event of various contingencies are defined by a formula while the contributions are to be determined by the actuary by means of the valuation process. The funding method then represents the means by which the contribution rate is fixed at each valuation. We shall consider the case of annual valuations: at which the actuary values the prospective liabilities, allowing for future contributions to be paid, and compares this result with the value of the assets currently held.

The paper provides a natural follow-up to the earlier work of DUFRESNE (1988) and HABERMAN (1992) and gives a comparison with these earlier results.

As in these earlier papers, we shall consider the funding methods described by the following pairs of equations:

$$
C(t)=N C(t)+A D J(t)
$$

where $C(t)$ is the contribution rate at time $t, N C(t)$ is the normal cost at time $t$ and $A D J(t)$ is an adjustment to the contribution rate at time $t$, represented by the liquidation of the unfunded liability, $U L(t) . U L(t)$ is defined by:

$$
U L(t)=A L(t)-F(t)
$$

ASTIN BULJ.ETIN. 1995, Vol. 25, No. 2, 177-187 
where $A L(t)$ is the total actuarial liability in terms of all members at time $t$ and $F(t)$ is the fund level at time $t$, measured in terms of the market value of the underlying assets.

We are using a discrete time approach with $t$ taking integer values $0,1,2$ and so on.

At each time $t$, a valuation is carried out to estimate $C(t)$ and $F(t)$ based on the membership of the scheme at that time. As $t$ changes, however, we allow for new entrants to the membership so that the population remains stationary. See the assumptions listed below.

In the ensuing mathematical discussion, we make the following assumptions:

1. All actuarial assumptions are consistently borne out by experience, except for investment returns.

2. The population is stationary in size and structure from the start.

3. Salaries increase at a deterministic rate of inflation. For simplicity, each active member's annual salary is set at 1 unit at the minimum age at entry. There is no promotional salary scale. We allow for salary inflation by considering the real rate of investment return i.e. the rate in excess of salary inflation. In parallel, we assume that benefits in payment increase at the same rate of salary inflation.

4. The real interest rate assumption for valuation purposes is fixed.

5. It is assumed that the contribution income and benefit outgo occur at the start of each scheme year.

It is straightforward to relax some of these assumptions e.g. replace 2 by allowing the population to grow at a fixed compound rate (i.e. be stable in the sense of KEYFITZ (1985)); include a promotion salary scale in 3; use a different timing assumption in 5 .

Assumptions 1.-4. imply that the following are constants with respect to time, $t$ (after rescaling to allow for the predetermined growth in line with salary inflation):

$N C$ : the total normal cost.

$A L$ : the total actuarial liability.

$B$ : the overall benefit outgo per unit time.

Also, assumptions 1., 2., 4. and 5. imply that the following equation of equilibrium holds :

$$
A L=(1+i)(A L+N C-B)
$$

or equivalently

$$
B=d . A L+N C \text { where } d=i(1+i)^{-1},
$$

the compound interest discount rate.

This equation of equilibrium can be also found in the earlier papers of Trowbridge (1952) and Bowers et al. (1976).

We make the following further assumptions regarding the real interest rate earned on the fund and the stochastic nature of $F(t)$ :

6. The real interest rate earned on the fund during the period $(t, t+1)$ is $i(t+1)$, where $E i(t+1)=i$, the real valuation rate of interest. Thus, the valuation rate is correct "on average". This assumption is not essential mathematically but is in agreement with classical ideas on pension fund valuation. We further define $\sigma^{2}=$ $\operatorname{Var} i(t+1)$. 
7. It is assumed that the earned real rates of return $i(t)$ for $t>1$ are independent, identically distributed random variables (with $i(t)>-1$ with probability 1).

8. $\operatorname{Prob}\left[F(O)=F_{0}\right]=1$ for some $F_{0}$.

Given these assumptions, the random variable $i(t)$ leads to $F(t)$ being a random variable and hence $U L(t), A D J(t)$ and $C(t)$ being random variables.

A continuous time formulation would be possible, in which case stochastic differential equations wold be utilised in the mathematical discussion rather than difference equations.

We are not suggesting (through assumption 7) that the rates of return actually achieved by pension funds form an independent and identically distributed sequence. Indeed, rates of return are more generally viewed as autoregressivemoving average processes (for example, PANJER and BELlHOUSE (1980)). In parallel work, HaBERMAN (1991, 1993) has investigated the effect of using dependent investment return models, in particular autoregressive models of low order. These more sophisticated models are not pursued here. It is only because it keeps the mathematical discussion tractable that assumption 7 is imposed here.

CHOICE OF $A D J$ : SPREAD PERIOD AND DELAY

We consider a particular method for defining the contribution adjustment term $A D J(t)$ which is an approach widely used in the U.K. and involves putting $A D J(t)$ equal to the overall unfunded liability divided by the present value of an annuity for a term of $M$ years, calculated at the valuation rate of interest $i$. It is common practice to use values of $M$ in the range 20-25 years, on the grounds that this would represent the average remaining active lifetime within the scheme of the current membership.

As in Haberman (1992), we shall allow for delays in the collection and processing of data and the preparation of the accounts, and assume that the adjustment term at time $t$ depends on $U L(t-1)$. Thus, with $\left.k=1 / \ddot{a}_{M}\right\rceil$,

$$
A D J(t)=k . U L(t-q) \text { where } q=0 \text { or } q=1 .
$$

$q=0$ corresponds to the analysis of DUFRESNE (1988) and $q=1$ corresponds to HABERMAN (1992). Then:

$$
C(t)=N C+k \cdot(A L-F(t-q)) \text { where } q=0 \text { or } q=1 \text {. }
$$

We shall now view $k$ (and hence $M$ ) and $q$ as being control parameters which the actuary may choose with the objective of meeting certain specified criteria (see later) connected with controlling the behaviour of $C(t)$ or $F(t)$ over time. The choice of $M$ would not be completely free : $M$ would probably have a lower bound to limit the income tax deductibility of contributions and an upper bound to prevent large increases in $U L(t)$.

Equation (5) includes a negative feedback component, whereby the current status is compared with a target and corrective action is taken to deal with the discrepancy.

With $q=1$ in equation (4) we see that an element of delay is introduced into the way that changes in $F($.$) feed back into changes in C($.$) .$ 
Other values of $q$ are considered in detail in ZIMBIDIS and HABERMAN (1993) and are not discussed here.

We note that this choice of $A D J(t)$ uses the same fraction of the unfunded liability regardless of the latter's sign, so surpluses and deficiencies are treated in the same way, which would not always be the case in practice.

\section{MOMENTS OF $F(t)$ and $C(t)$}

In the case of $q=0$, we repeat from DUFRESNE (1988) the recurrence relation for $F(t)$ :

$$
F(t+1)=[u(t+1) / u](p F(t)+r)
$$

and from HABERMAN (1992) the corresponding relation when $q=1$

$$
F(t+1)=[u(t+1) / u](u F(t)-u k F(t-1)+r)
$$

where we have introduced the subsidiary parameters

$$
u=(1+i), p=(1+i)(1-k), r=(1+i)(N C-B+k A L)
$$

and

$$
u(t+1)=1+i(t+1) .
$$

Using conditional expectation and variance based methods, DUFRESNE (1988) obtains explicit equations for the expectation and variance of $F(t)$ and $C(t)$ for finite $t$ when $q=0$. In the limit as $t \rightarrow \infty$, he demonstrates that, providing that $M>1$,

$$
\lim _{t \rightarrow \infty} E F(t)=A L
$$

and

$$
\lim _{t \rightarrow \infty} E C(t)=N C
$$

and that providing that $y(1-k)^{2}<1$

$$
\lim _{t \rightarrow \infty} \operatorname{Var} F(t)=\frac{\sigma^{2} A L^{2}}{u^{2}\left(1-y(1-k)^{2}\right)}
$$

where $y=\sigma^{2}+u^{2}$, and

$$
\lim _{t \rightarrow \infty} \operatorname{Var} C(t)=\frac{\sigma^{2} k^{2} A L^{2}}{u^{2}\left(1-y(1-k)^{2}\right)} .
$$

In this discussion, we exclude pay-as-you-go funding and terminal funding for which $A L=0$ and initial funding. 
Using conditional expectation and generating function based methods, HABERMAN (1992) similarly obtain equations for the first two moments of $F(t)$ and $C(t)$ when $q=1$. In the limit as $t \rightarrow \infty$, he demonstrates that

$$
\begin{aligned}
& \lim _{t \rightarrow \infty} E F(t)=A L \\
& \lim _{t \rightarrow \infty} E C(t)=N C
\end{aligned}
$$

providing that $M \geq 2$ and $a_{M\rceil}>1$.

Under more complex restrictions on the parameters (Appendix I), HABERMAN (1992) obtains, for the case $q=1$, that

$$
\lim _{t \rightarrow \infty} \operatorname{Var} F(t)=\frac{\sigma^{2} A L^{2}(1+u k)}{u^{2}\left(1+k u-y\left(1-u k+k^{2}+u k^{3}\right)\right)}
$$

and

$$
\lim _{t \rightarrow \infty} \operatorname{Var} C(t)=\frac{\sigma^{2} k^{2} A L^{2}(1+u k)}{u^{2}\left(1+k u-y\left(1-u k+k^{2}+u k^{3}\right)\right)}
$$

[Note that there is a typographical error in equations (14), (15) and (B.6) in HABERMAN (1992)].

\section{TRADE OFF IN VARIANCES}

We introduce the following notation for the scaled variances

and

$$
\left.\begin{array}{l}
\alpha_{i}(M)=\frac{\lim \operatorname{Var} F(t)}{(\lim E F(t))^{2}} \\
\beta_{i}(M)=\frac{\lim \operatorname{Var} C(t)}{(\lim E C(t))^{2}}
\end{array}\right\} \text { for } q=i \text { where } i=0 \text { or } 1
$$

Then Dufresne has shown that, if $y>1$, then there exists $M^{*}$ such that i) for $M \leq M^{*}, \alpha_{0}(M)$ increases and $\beta_{0}(M)$ decreases with increasing $M$,

ii) for $M>M^{*}$, both $\alpha_{0}(M)$ and $\beta_{0}(M)$ increase with increasing $M$ and that

$$
\ddot{a}_{M *\rceil}=\frac{1}{k^{*}} \text { where } k^{*}=1-\frac{1}{y} .
$$

In a sense, the choice of $M$ in the range of $\left(1, M^{*}\right)$ is "optimal". If our objective in choosing $M$ is to reduce uncertainty and to keep the limiting variances of $F(t)$ and $C(t)$ to a minimum, then any $M>M^{*}$ is to be discarded since clearly some 
other $M<M^{*}$ would at least reduce $\alpha_{0}(M)$ while keeping $\beta_{0}(M)$ the same. For most pension funds, it is likely that the variance of $C(t)$ will be the principal criterion of interest.

We shall now consider the extent to which similar properties hold for the case $q=1$.

We firstly note that, for $M>1$,

$$
\left.\begin{array}{l}
\alpha_{0}(M)<\alpha_{1}(M) \\
\text { and } \\
\beta_{0}(M)<\beta_{1}(M)
\end{array}\right\}
$$

(as demonstrated in the numerical examples in HABERMAN (1992)).

The proof is straightforward. We consider

$$
\begin{aligned}
\frac{\alpha_{1}(M)}{\alpha_{0}(M)}=\frac{\beta_{1}(M)}{\beta_{0}(M)} & =\frac{(1+u k)\left(1-y(1-k)^{2}\right)}{1+u k-y\left(1-k u+k^{2}+u k^{3}\right)} \\
& =\frac{1-y(1-k)^{2}}{1-y\left(1-k u+k^{2}+u k^{3}\right) /(1+u k)} \\
& =\frac{1-y\left(1+k^{2}-2 k\right)}{1-y\left(1+k^{2}-2 u k /(1+u k)\right)}
\end{aligned}
$$

The difference between the terms in the numerator and denominator is the coefficient of " $2 k$ ". Now, if $M>1$,

$$
\begin{gathered}
\frac{u}{1+u k}<1 \text { because } 1+u k-u=\frac{1}{\left.a_{M}\right\rceil}-i>0 . \\
\text { Hence } \frac{\alpha_{1}(M)}{\alpha_{0}(M)}=\frac{\beta_{1}(M)}{\beta_{0}(M)}>1 .
\end{gathered}
$$

(HABERMAN and ZimBIDIS (1993) show that these inequalities hold for higher values of $q$ ). This result is intuitive: the introduction of a one year time delay means that we have lost information about the fund since time $t-1$ and we would expect the resulting variances to be increased.

We consider the behaviour of $\alpha_{1}(M)$ as $M$ varies. It is convenient to view $\alpha_{1}()$ as a function of $k$ and then use the 1-1 correspondence between values of $k$ and $M$.

We can show that

$$
\frac{d}{d k} \alpha_{1}(k)=\frac{\sigma^{2} 2 y\left(k(1+k u)^{2}-u\right)}{u^{2}\left(1+k u-y\left(1-u k+k^{2}+u k^{3}\right)\right)^{2}} .
$$

We are interested in the turning points of $\alpha_{1}(k)$ in the range for $k$ of $(d, 1)$, corresponding to values of $M$ in the range $(1, \infty)$. 
The cubic equation $p(k)=k(1+k u)^{2}-u$ has only one real root since

$$
D=\frac{1}{u^{4}}\left(27 u^{2}+4\right)>0
$$

(see Appendix II). We let $k_{1}$ be this real root.

We note that $p(d)=u(i-1)<0$ (if $|i|<100 \%$ ) and $p(1)=1+u+u^{2}>0$.

Then $p(k)<0$ for $d<k<k_{1}$ and $p(k)>0$ for $k_{1}<k<1$.

$k_{1}$ depends on the value of $u$ and numerical experiments indicate the following values:

\begin{tabular}{clc}
\hline$i$ & $u$ & $k_{1}$ \\
\hline 0 & 1 & 0.4656 \\
$1 \%$ & 1.01 & 0.4666 \\
$5 \%$ & 1.05 & 0.4704 \\
$10 \%$ & 1.10 & 0.4707 \\
$20 \%$ & 1.20 & 0.4818 \\
\hline
\end{tabular}

In each of the cases, $k_{1}$ approximately corresponds to $M_{1}=2$.

Hence

$$
\frac{d}{d k} \alpha_{1}(k)<0 \text { for } d<k<k_{1}
$$

and

$$
\frac{d}{d k} \alpha_{1}(k)>0 \text { for } k_{1}<k<1
$$

(subject to $k$ satisfying the constraints implied by Appendix I) which are equivalent to

$$
\frac{d}{d M} \alpha_{1}(M)<0 \text { for } 1<M<M_{1}=2
$$

and

$$
\frac{d}{d M} \alpha_{1}(M)>0 \text { for } M_{1}<M<\infty .
$$

We now consider the behaviour of $\beta_{1}(k)$, viewed initially as a function of $k$. We can show that

$$
\frac{d}{d k} \beta_{1}(k)=\frac{\sigma^{2} 2 k\left(1-y+k u(2-y)+u^{2} k^{2}(1+y)\right)}{u^{2}\left(1+k u-y\left(1-u k+k^{2}+u k^{3}\right)\right)^{2}}
$$


We are interested in the turning points of $\beta_{1}(k)$ in the feasible range $k$ of $(d, 1)$, again subject to the constraints implied by Appendix I, corresponding to values of $M$ in the range $(1, \infty)$.

The quadratic equation $s(k)=1-y+k u(2-y)+u^{2} k^{2}(1+y)$ has one real root in the specified range since $y>0.8$. We let $k_{2}$ be this real root.

We note that

$s(0)=1-y<0$

and

$$
\begin{aligned}
& s(1)=1-y+u(2-y)+u^{2}(1+y)= \\
& u^{3}(u-1)+2 u+1+\sigma^{2}\left(u^{2}-u-1\right)
\end{aligned}
$$

so

$$
s(1)>0 \text { if } i>61.8 \% \text {, given that } u>0
$$

or

$$
s(1)>0 \text { if } \sigma^{2}<\frac{u^{3}(u-1)+2 u+1}{1+u-u^{2}}=\sigma_{1}^{2} \text {, say. }
$$

[This restriction on $\sigma^{2}$ is not too onerous! It would correspond to the following values:

\begin{tabular}{cc}
\hline \multicolumn{1}{c}{$i$} & \multicolumn{1}{c}{$\sigma_{1}^{2}$} \\
\hline 0 & $300 \%$ \\
$1 \%$ & $306 \%$ \\
$5 \%$ & $333 \%$ \\
$10 \%$ & $375 \%$ \\
$20 \%$ & $493 \%]$ \\
\hline
\end{tabular}

Clearly $k_{2}$ corresponds to a minimum value of $\beta(k)$.

The explicit value of $k_{2}$ is

$$
k_{2}=\frac{-(2-y)+\sqrt{y(5 y-4)}}{2 u(1+y)}
$$

Thus

$$
\frac{d}{d k} \beta_{1}(k)<0 \text { for } d<k<k_{2}
$$

and

$$
\frac{d}{d k} \beta_{1}(k)>0 \text { for } k_{2}<k<1 .
$$

If $k_{2}$ corresponds to $M_{2}$ we can translate this statement into

$$
\frac{d}{d M} \beta_{1}(M)<0 \text { for } 1<M<M_{2}
$$


and

$$
\frac{d}{d M} \beta_{1}(M)>0 \text { for } M_{2}<M<\infty .
$$

Given the restrictions on the parameters mentioned in the above discussion, we see that for the case of $q=1$ that is also a trade off between variability in $C(t)$ and variability in $F(t)$ and that there is an optimal choice of $M$, and hence of $k$, if our objective is to keep the limiting variances to a minimum. The optimal spread period in this case is $\left(1, M_{2}\right)$.

A comparison of the optimal periods defined by $M^{*}$ and $M_{2}$ (for the cases $q=0$ and $q=1$ ) would be useful. Again it is convenient to examine the corresponding annuity values, as represented by $k$.

We note that

$$
\begin{aligned}
s^{\prime}\left(k^{*}\right) & =u(2-y)+2 u^{2}(1+y) \frac{(y-1)}{y} \\
& =\frac{u}{y}\left[2\left(\sigma^{2}+u^{2}-u\right)+y^{2}(2 u-1)\right] .
\end{aligned}
$$

The sign of $s^{\prime}\left(k^{*}\right)$ depends on the values of $u$ and $\sigma$. Clearly, if $u \geq 1$ (i.e. $i \geq 0$ ) then $s^{\prime}\left(k^{*}\right)>0$. Since $s(k)$ is a quadratic, with minimum at $k=k_{2}$, this implies that $k^{*}>k_{2}$ and hence that $M_{2}>M^{*}$ (for $i \geq 0$ ).

This is confirmed by the numerical example given in HABERMAN (1992). As Table 1 illustrates, the values of $M_{2}$ and $M^{*}$ are numerically close.

\begin{tabular}{|c|c|c|c|c|c|c|}
\hline \multirow[b]{2}{*}{$\sigma$} & \multicolumn{6}{|c|}{ Real valuation rate of interest } \\
\hline & 0 & 0.01 & & & & \\
\hline & $M^{*}$ and $M_{2}$ & $M^{*}$ and $M_{2}$ & $M^{*}$ & $M_{2}$ & $M^{*}$ & $M_{2}$ \\
\hline 0.05 & 401 & 60 & 23 & 24 & 14 & 15 \\
\hline 0.10 & 101 & 42 & 20 & 20 & 13 & 14 \\
\hline 0.25 & 45 & 28 & 16 & 17 & 11 & 12 \\
\hline 0.20 & 26 & 19 & 13 & 13 & 10 & 10 \\
\hline 0.25 & 17 & 14 & 10 & 11 & 8 & 9 \\
\hline
\end{tabular}

TABLE 1

Numerical Values of $M^{*}$ AND $M_{2}$

CONCLUSIONS

A simple stochastic model is used to represent the real investment returns for a defined benefit pension scheme. 
The paper shows that in the presence of a one year time delay between fixing a contribution rate and the information about current fund levels, it is possible to set up formulae for studying the limiting behaviour of the expected values and variances of the contributions and fund levels. The paper demonstrates that, as with the case when there is no time delay, there is an optimum range of values for the spread period, $M$ (for amortizing valuation surpluses or deficiencies). The relationship between the optimum range of values of $M$ in the case of no time delay $(q=0)$ and with a one year time delay $(q=1)$ is investigated.

\section{ACKNOWLEDGEMENT}

This research work was performed under EEC contract SPES-CT91-0063.

\section{Appendix I}

Based on HABERMAN (1992) Appendix C, we require the following conditions for the convergence of equations $(10)$ :

With $b=\sigma^{2}+u^{2}-u k$

$$
\begin{aligned}
& c=\left(\sigma^{2}+u^{2}\right) k(u-k) \\
& e=\left(\sigma^{2}+u^{2}\right) u k^{3} \\
& D=27 e^{2}+4 c^{3}-18 b c e-b^{2} c^{2}+4 b^{3} e,
\end{aligned}
$$

we require

i) $1+c>|b+e|$

ii) (a) if $D>0, e^{3}-b e+c-1<0$

(b) if $D<0,|b|<\frac{1}{2}(3+c)$.

i) and ii) can be considered to provide restrictions on $k$ (and hence $M$ ) or on $\sigma^{2}$.

\section{Appendix II - Roots of a cubic equation}

In general, the roots of the cubic equation

$$
p(x)=x^{3}-b x^{2}+c x-e
$$

are

$$
\begin{gathered}
x_{1}=\frac{1}{3} b+U+V \\
x_{2}, x_{3}=\frac{1}{3} b-\frac{1}{2}(U+V) \pm \frac{\sqrt{3}}{2} i(U-V)
\end{gathered}
$$


where

$$
U, V=\left[\frac{b^{3}}{27}-\frac{b c}{6}+\frac{e}{2} \pm \frac{1}{6 \sqrt{3}} \sqrt{D}\right]
$$

and

$$
D=27 e^{2}+4 c^{3}-18 b c e-b^{2} c^{2}+4 b^{3} e .
$$

\section{REFERENCES}

Bowers, N. L., Hickman, J. C. and Nesbitt, C. J. (1976) Introduction to the dynamics of pension funding. Transactions of the Society of Actuaries, 28, 177-203.

Dufresne, D. (1988) Moments of pension fund contributions and fund levels when rates of return are random. Journal of the Institute of Actuaries, 115, 535-544.

Haberman, S. (1991) Pension funding methods and autoregressive interest rate models. Proceedings of Second AFIR Colloquium. Volume 2, pp. 181-204.

Haberman, S. (1992) Pension funding with time delays: a stochastic approach. Insurance: Mathematics and Economics, 11, 179-189.

Haberman, S. (1993) Pension funding with time delays and autoregressive rates of investment return. Insurance: Mathematics and Economics, 13, 45-56.

Keyfitz, N. (1985) Applied Mathematical Demography. (2nd edition). Springer Verlag, New York.

PANJer, H.H. and Bellhouse, D. R. (1980) Stochastic modelling of interest rates with applications to life contingencies. Journal of Risk and Insurance, 47, 91-110.

Trowbridge, C. L. (1952) Fundamentals of pension funding. Transactions of the Society of Actuaries, 4, $17-43$.

Zimbidis, A. and HabERman, S. (1993) Delay, feedback and the variability of pension contributions and fund levels. Insurance: Mathematics and Economics, 13, 271-285.

Steven Haberman

Department of Actuarial Science and Statistics

The City University

Northampton Square

London ECIV GHB

$U K$. 